Pacific Journal of Mathematics

ON THE SPECTRUM OF A TOEPLITZ OPERATOR 


\section{ON THE SPECTRUM OF A TOEPLITZ OPERATOR}

\section{HAROLD WIDOM}

Given a function $\phi \in L_{\infty}(-\pi, \pi)$, the Toeplitz operator $T_{\phi}$ is the operator on $H_{2}$ (the set of $f \in L_{2}$ with Fourier series of the form $\sum_{0}^{\infty} c_{n} e^{i n \theta}$ ) which consists of multiplication by $\phi$ followed by $P$, the natural projection of $L_{2}$ onto $H_{2}$ : if $f \sim \sum_{-\infty}^{\infty} c_{n} e^{i n \theta}$ then $P f \sim \sum_{0}^{\infty} c_{n} e^{i n \theta}$. Succinctly,

$$
T_{\phi} f=P(\phi f)
$$

$f \in H_{2}$.

In [5] a necessary and sufficient condition on $\phi$ was given for the invertibility of $T_{\phi}$. This will be stated below. (The paper [5] is needlessly complicated. In a recent paper of Devinatz [1], however, all results of [5] and more are proved without undue complication in a general Dirichlet algebra setting.) Halmos [2] has posed the following as a test question for any theory of invertibility of Toeplitz operators: Is the spectrum of a Toeplitz operator necessarily connected? We shall shown here that the answer is Yes.

The proof consists mainly of applications of Theorem I of [5], which says the following.

A necessary and sufficient condition for the invertibility of $T_{\phi}$ is the existence of function $\phi_{+}$and $\phi_{-}$belonging respectively to $H_{2}$ and $\bar{H}_{2}$ (the set of complex conjugates of $H_{2}$ functions) such that

(a) $\phi=\phi_{+} \phi_{-}$,

(b) $\phi_{+}^{-1} \in H_{2}$ and $\phi_{-}^{-1} \in \bar{H}_{2}$,

(c) for $f \in L_{\infty}, \quad S f=\phi_{+}^{-1} P \phi_{-}^{-1} f \in L_{2}$, and $f \rightarrow S f$ extends to a bounded operator on $L_{2}$.

We don't want to prove the theorem here but we do have to say where the functions $\phi_{ \pm}$come from under the assumption that $T_{\phi}$ is ivertible. If we set

$$
\psi_{+}=T_{\phi}^{-1} 1, \bar{\psi}_{-}=T_{\phi}^{*-1} 1
$$

then it can be shown that $\phi \psi_{+} \psi_{-}=c$, a constant. We must have $c \neq 0$ since $\psi_{ \pm}$can vanish only on sets of measure zero and $\phi$ is not identically zero. One then defines

$$
\phi_{+}=1 / \psi_{+}, \quad \phi_{-}=c / \psi_{-}
$$

and (a) and (b) hold.

As for the relevance of condition (c), it turns out that the ex-

Received April 15, 1963. Sloan Foundation fellow. 
tension of $S$, restricted to $H_{2}$, is exactly $T_{\phi}^{-1}$. It follows that

$$
\left\|(P f) \phi_{-}\right\|_{2} \leqq\|\phi\|_{\infty}\left\|T_{\phi}^{-1}\right\|\left\|f \phi_{-}\right\|_{2} \quad f \in L_{\infty} .
$$

Conversely, suppose there exists an $M$ such that

$$
\left\|(P f) \phi_{-}\right\|_{2} \leqq M\left\|f \phi_{-}\right\|_{2} \quad f \in L_{\infty} \text {. }
$$

Then we can deduce

$$
\left\|\phi_{+}^{-1} P \phi_{-}^{-1} f\right\|_{2} \leqq M\left\|\phi^{-1}\right\|_{\infty}\|f\|_{2}
$$

It is a simple consequence of (c) that $\left\|\phi^{-1}\right\|_{\infty}<\infty$. (See [5], Theorem I, corollary, or [1], Lemma 2.) Thus (c) may be replaced by

(c') $\phi^{-1} \in L_{\infty}$ and the map $f \rightarrow P f$ is bounded in the space $L_{2}\left(\left|\phi_{-}\right|^{2} d \theta\right)$.

We shall need this fact.

To begin the proof of the connectedness of $\sigma\left(T_{\phi}\right)$, the spectrum of $T_{\phi}$, let $\Lambda$ be a compact set disjoint from $\sigma\left(T_{\phi}\right)$. (Think of $\Lambda$ as being a simple closed curve surrounding a portion of $\sigma\left(T_{\phi}\right)$.) For each $\lambda \in \Lambda$ the operator $T_{\phi}-\lambda=T_{\phi-\lambda}$ is invertible, so we have the corresponding functions

$$
\psi_{+}(\lambda)=\left(T_{\phi}-\lambda\right)^{-1} 1, \quad \bar{\psi}_{-}(\lambda)=\left(T_{\phi}-\lambda\right)^{*-1} 1
$$

and the constant $c(\lambda)$ as described above, and

$$
\phi-\lambda=\phi_{+}(\lambda) \phi_{-}(\lambda)
$$

where

$$
\phi_{+}(\lambda)=1 / \psi_{+}(\lambda), \quad \phi_{-}(\lambda)=c(\lambda) / \psi_{-}(\lambda) .
$$

Let us consider the continuity of these various function of $\lambda$. It follows from the definition of $\psi_{ \pm}(\lambda)$ and the continuity, in the uniform operator topology, of the mappings $\lambda \rightarrow\left(T_{\phi}-\lambda\right)^{-1}$ and $\lambda \rightarrow\left(T_{\phi}-\lambda\right)^{*-1}$, that $\lambda \rightarrow \psi_{ \pm}(\lambda)$ are continuous functions from $\Lambda$ to $L_{2}$. This implies that $\lambda \rightarrow c(\lambda) /(\phi-\lambda)$ is continuous from $\Lambda$ to $L_{1}$. Since $\lambda \rightarrow \phi-\lambda$ is continuous from $\Lambda$ to $L_{\infty}$ we conclude that $\lambda \rightarrow c(\lambda)$ is continuous from $\Lambda$ to $L_{1}$, so $c(\lambda)$ is a continuous complex valued function. Since $c(\lambda) \neq 0$ it follows also that $\lambda \rightarrow \phi_{+}(\lambda)=(\phi-\lambda) \psi_{-}(\lambda) / c(\lambda)$ and $\lambda \rightarrow \phi_{-}(\lambda)=(\phi-\lambda) \psi_{+}(\lambda)$ are continuous from $\Lambda$ to $L_{2}$. To recapitulate, the four functions $\phi_{ \pm}(\lambda)^{ \pm 1}$ are $L_{2}$ continuous.

The next step is to take logarithms. Since both $\phi_{+}(\lambda)$ and $1 / \phi_{+}(\lambda)$ belong to $H_{2}, \phi_{+}(\lambda)$ is an outer function. Recall that this means it has the representation

$$
\phi_{+}(\lambda)=\alpha_{+}(\lambda) e^{\log \left|\phi_{+}(\lambda)\right|+i\left[\log \left|\phi_{+}(\lambda)\right|\right]^{\sim}}
$$


where the tilde denotes conjugate function and

$$
\alpha_{+}(\lambda)=\operatorname{sgn} \int \phi_{+}(\lambda) d \theta
$$

is a constant of absolute value 1 . Since $\phi_{+}(\lambda)^{ \pm 1}$ are $L_{2}$ continuous so is $\log \left|\phi_{+}(\lambda)\right|$, and therefore also $\left[\log \left|\phi_{+}(\lambda)\right|\right]^{\sim}$ (since $u \rightarrow \widetilde{u}$ is $L_{2}$ continuous). The continuity of the complex valued function $\alpha_{+}(\lambda)$ follows from the fact that $\int \phi_{+}(\lambda) d \theta$ is continuous and nonzero.

Similarly we can write

$$
\phi_{-}(\lambda)=\alpha_{-}(\lambda) e^{\log \left|\phi_{-}(\lambda)\right|-i\left[\log \left|\phi_{-}(\lambda)\right|\right]^{\sim}}
$$

with $\alpha_{-}(\lambda)$ continuous and nonzero. Putting our representations together and using (2) we have

$$
\phi-\lambda=\alpha(\lambda) e^{\log \left|\phi_{+}(\lambda)\right|+i\left[\log \left|\phi_{+}(\lambda)\right|\right]^{\sim}} e^{\operatorname{lng}\left|\phi_{-}(\lambda)\right|-i\left[\log \left|\phi_{-}(\lambda)\right|\right]^{\sim}}
$$

where $\alpha(\lambda)=\alpha_{+}(\lambda) \alpha_{-}(\lambda)$ is a continuous nowhere vanishing complex valued function.

The sum of the two exponents in (3), which we shall call $l(\lambda, \theta)$, is for each $\lambda$ an element of $L_{2}$, and the map $\lambda \rightarrow l(\lambda, \cdot)$ is $L_{2}$ continuous. It is important that we be able to say that for each $\theta$ (or almost every $\theta), l(\lambda, \theta)$ is a continuous function of $\lambda$. This is false for general $L_{2}$ valued functions but in our situation something as good is true.

Lemma 1. There is a null set $N \subset(-\pi, \pi)$ and a function $L(\lambda, \theta)$ defined on $\Lambda \times N^{\prime}$ such that for each $\lambda$

$$
L(\lambda, \theta)=l(\lambda, \theta) a . e .,
$$

for each $\theta \in N^{\prime}$

$$
L(\lambda, \theta) \text { is continuous in } \lambda \text {. }
$$

and for all $\lambda \in \Lambda, \theta \in N^{\prime}$

$$
\phi(\theta)-\lambda=\alpha(\lambda) e^{L(\lambda, \theta)} .
$$

Proof. First we make sure that $\phi$ is defined everywhere and that its range has positive distance from $\Lambda$. This we can do since $\Lambda$ is a compact set disjoint from $R(\phi)$, the essential range of $\phi$. (Recall that $T_{\phi-\lambda}$ invertible implies $(\phi-\lambda)^{-1} \in L_{\infty}$.)

Take $\lambda_{0} \in \Lambda$ and let $L_{0}\left(\lambda_{0}, \theta\right)$ be a function of $\theta$ which equals $l\left(\lambda_{0}, \theta\right)$ a.e. and for which

$$
\phi(\theta)-\lambda_{0}=\alpha\left(\lambda_{0}\right) e^{\underline{L}_{0}\left(\lambda_{0}, \theta\right)}
$$


everywhere. Let $U=\left\{\lambda \in \Lambda:\left|\lambda-\lambda_{0}\right|<\delta\right\}$ be a neighborhood of $\lambda_{0}$ so small that $\lambda \in U$ implies

$$
\begin{aligned}
& \left|\frac{\alpha(\lambda)}{\alpha\left(\lambda_{0}\right)}-1\right|<1, \\
& \left|\frac{\phi(\theta)-\lambda}{\phi(\theta)-\lambda_{0}}-1\right|<1,
\end{aligned}
$$

We extend $L_{0}\left(\lambda_{0} \theta\right)$ to a function defined on $U \times(-\pi, \pi)$ by

$$
L_{0}(\lambda, \theta)=L_{0}\left(\lambda_{0}, \theta\right)+\log \frac{\phi(\theta)-\lambda}{\phi(\theta)-\lambda_{0}}-\log \frac{\alpha(\lambda)}{\alpha\left(\lambda_{0}\right)}
$$

where the logarithms are defined by the usual power series. Clearly $L_{0}(\lambda, \theta)$ is continuous on $U$ for each $\theta$ and $\phi(\theta)-\lambda=\alpha(\lambda) e^{L_{0}(\lambda, \theta)}$ everywhere on $U \times(-\pi, \pi)$. We shall show $L_{0}(\lambda, \theta)=l(\lambda, \theta)$ a.e. for each $\lambda \in U$, at least if $\delta$ is small enough. Let us set

$$
\begin{aligned}
& u_{+}(\lambda)=\frac{\phi_{+}(\lambda)}{\alpha_{+}(\lambda)}=e^{\log \left|\phi_{+}(\lambda)\right|+i\left[\log \left|\phi_{+}(\lambda)\right|\right]^{\sim}} \\
& u_{-}(\lambda)=\frac{\phi_{-}(\lambda)}{\alpha_{-}(\lambda)}=e^{\log \left|\phi_{-}(\lambda)\right|-i\left[\log \left|\phi_{-}(\lambda)\right|\right]^{\sim}}
\end{aligned}
$$

and

$$
v_{ \pm}(\lambda)=e^{1 / 2 L_{0}(\lambda \theta) \pm i / 2 \tilde{L}_{0}(\lambda, \theta)} .
$$

We know $u_{+}(\lambda)^{ \pm 1} \in L_{2}$. Actually for each $\lambda, u_{+}(\lambda)^{ \pm 1} \in L_{p}$ for some $p>2$ (the $p$ depending on $\lambda$ ). The reason is the following. Condition $\left(c^{\prime}\right)$ in the criterion given above for invertibility implies that the map $f \rightarrow P f$ is bounded in the space $L_{2}\left(\left|u_{-}(\lambda)\right|^{2} d \theta\right)$. Helson and Szegö have determined ([3], Theorem 1) all measures $d \mu$ such that $f \rightarrow P f$ is bounded in $L_{2}(d \mu)$. They are measures of the form

$$
d \mu=e^{\rho+\tilde{\sigma}} d \theta
$$

with $\rho \in L_{\infty}$ and $\|\sigma\|_{\infty}<\pi / 2$. However

$$
\|\sigma\|_{\infty}<\frac{\pi}{2} \text { implies } e^{\tilde{\sigma}} \in L_{1} \text {. }
$$

This is a theorem of Zygmund. (See [6], p. 257.) A statement. which is only at first glance stronger is

$$
\|\sigma\|_{\infty}<\frac{\pi}{2} \text { implies } e^{ \pm \tilde{\sigma}} \in L_{1+\varepsilon} \text { for some } \varepsilon>0 .
$$

Putting these things together we can conclude that $u_{-}(\lambda)^{ \pm 1} \in L_{p}$ for 
some $p>2$, and so also $u_{+}(\lambda)^{ \pm 1} \in L_{p}$.

Since $L_{0}\left(\lambda_{0}, \theta\right)=l\left(\lambda_{0}, \theta\right)$ a.e., a routine check shows $\left|v_{+}\left(\lambda_{0}\right)\right|=$ $c\left|u_{+}\left(\lambda_{0}\right)\right|$ a.e., where $c$ is a nonzero constant, so we have $v_{+}\left(\lambda_{0}\right)^{ \pm 1} \in L_{p_{0}}$. We shall show from this that $v_{+}(\lambda)^{ \pm 1} \in L_{2}$ for all $\lambda \in U$ is $\delta$ is sufficiently small. We have

$$
\frac{v_{+}(\lambda)}{v_{+}\left(\lambda_{0}\right)}=e^{1 / 2\left[L_{0}(\lambda, \theta)-L_{0}\left(\lambda_{0}, \theta\right)\right]} e^{i / 2\left[\widetilde{L}_{0}(\lambda, \theta)-\widetilde{L}_{0}\left(\lambda_{0}, \theta\right)\right]}
$$

It follows from (4) that

$$
\lim _{\lambda \rightarrow \lambda_{0}}\left\|L_{0}(\lambda, \theta)-L_{0}\left(\lambda_{0}, \theta\right)\right\|_{\infty}=0 .
$$

Therefore, from Zygmund's theorem again, we can say this: given any $q_{0}<\infty$ there exists a $\delta$ so that $v_{+}(\lambda) / v_{+}\left(\lambda_{0}\right) \in L_{q_{0}}$ whenever $\left|\lambda-\lambda_{0}\right|<\delta$. If we choose $q_{0}$ so that $p_{0}^{-1}+q_{0}^{-1}=1 / 2$ then we shall have $v_{+}(\lambda) \in L_{2}$. In fact me shall have $v_{+}(\lambda) \in H_{2}$. (Any function of the form $\exp (\sigma+i \widetilde{\sigma}), \sigma \in L_{2}$, which belongs to $L_{2}$ also belongs to $H_{2}$; see [6], pp. 282-3.) Similarly

$$
v_{+}(\lambda)^{-1} \in H_{2} \text { and } v_{-}(\lambda)^{ \pm 1} \in \bar{H}_{2} \text {. }
$$

Now almost everywhere

$$
u_{+}(\lambda) u_{-}(\lambda)=v_{+}(\lambda) v_{-}(\lambda)\left(=\frac{\phi-\lambda}{\alpha(\lambda)}\right)
$$

so

$$
\frac{u_{+}(\lambda)}{v_{+}(\lambda)}=\frac{v_{-}(\lambda)}{u_{-}(\lambda)}
$$

The left side belongs to $H_{1}$ and the right to $\bar{H}_{1}$ so both sides must be a constant $\beta=\beta(\lambda)$, and

$$
\frac{v_{-}(\lambda)}{v_{+}(\lambda)}=\beta(\lambda)^{2} \frac{u_{-}(\lambda)}{u_{+}(\lambda)}
$$

If we take the logarithm of the absolute value of both sides we obtain

$$
\left[\mathscr{J} L_{0}(\lambda, \theta)\right]^{\sim}=2 \log |\beta(\lambda)|+\log \left|\phi_{-}(\lambda)\right|-\log \left|\phi_{+}(\lambda)\right|
$$

and so

$$
\mathscr{I} L_{0}(\lambda, \theta)=\left[\log \left|\phi_{+}(\lambda)\right|\right]^{\sim}-\left[\log \left|\phi_{-}(\lambda)\right|\right]^{\sim}+\gamma(\lambda)
$$

where $\gamma(\lambda)$ is, for each $\lambda$, a constant. Since

$$
\mathscr{R} L_{0}(\lambda, \theta)=\log \left|\frac{\phi(\theta)-\lambda}{\alpha(\lambda)}\right|=\log \left|\phi_{+}(\lambda)\right|+\log \left|\phi_{-}(\lambda)\right|
$$


we have upon adding,

$$
L_{0}(\lambda, \theta)=l(\lambda, \theta)+i \gamma(\lambda)
$$

Given a sequence $\lambda_{n} \rightarrow \lambda\left(\lambda_{n}, \lambda \in U\right)$ there is a subsequence $\lambda_{n^{\prime}}$ for which $l\left(\lambda_{n^{\prime}}, \theta\right) \rightarrow l(\lambda, \theta)$ a.e. (This follows from the $L_{2}$ continuity of l.) Since $L_{0}\left(\lambda_{n}, \theta\right) \rightarrow L_{0}(\lambda, \theta)$ everywhere we have $\gamma\left(\lambda_{n^{\prime}}\right) \rightarrow \gamma(\lambda)$. This shows that $\gamma$ is a continuous function of $\lambda$. Since $\gamma\left(\lambda_{0}\right)=0$ (recall that by definition, $L_{0}\left(\lambda_{0}, \theta\right)=l\left(\lambda_{0}, \theta\right)$ a.e.) and $\gamma$ is for each $\lambda$ an integral multiple of $2 \pi$, we must have $\gamma(\lambda)=0$. Thus $L_{0}(\lambda, \theta)=$ $l(\lambda, \theta)$ a.e. for each $\lambda \in U$.

Because of what we have done and the compactness of $A$ we can find a finite open covering $\left\{U_{k}\right\}$ of $\Lambda$ and for each $k$ a function $L_{k}(\lambda, \theta)$ defined on $U_{k} \times(-\pi, \pi)$ so that $L_{k}(\lambda, \theta)=l(\lambda, \theta)$ a.e. for each $\lambda \in U_{k}, L_{k}(\lambda, \theta)$ is continuous on $U_{k}$ for each $\theta$, and $\phi(\theta)-\lambda=$ $\alpha(\lambda) e^{L_{k}(\lambda, \theta)}$ on $U_{k} \times(-\pi, \pi)$. Consider a pair of these open sets $U_{j}$ and $U_{k}$, and let $\lambda_{1}, \lambda_{2}, \cdots$ be dense in $U_{j} \cap U_{k}$. For each $\lambda_{n}$ there is a $\theta$-set $E_{n}$ of measure zero outside of which both $L_{j}\left(\lambda_{n}, \theta\right)$ and $L_{k}\left(\lambda_{n}, \theta\right)$ equal $l\left(\lambda_{n}, \theta\right)$. Thus if $\theta$ does not belong to $\bigcup E_{n}$ we have $L_{j}\left(\lambda_{n}, \theta\right)=L_{k}\left(\lambda_{n}, \theta\right)$ for all $n$. By the continuity of $L_{j}$ and $L_{k}$ in $\lambda$ and the density of $\left\{\lambda_{n}\right\}$ we conclude that $L_{j}(\lambda, \theta)=L_{k}(\lambda, \theta)$ for all $\lambda \in U_{j} \cap U_{k}$ as long as $\theta$ does not belong to the set $F_{j, k}=\bigcup E_{n}$. Thus as long as $\theta$ does not belong to the set $N=\bigcup_{j, k} F_{j, k}$ any two of the functions $L_{k}(\lambda, \theta)$ agree where they are both defined. We can therefore combine all the $L_{k}$ to define a single function $L(\lambda, \theta)$ on $\Lambda \times N^{\prime}$ which has all the required properties.

LEMMA 2. If $\Lambda$ is a simple closed curve disjoint from $\sigma\left(T_{\phi}\right)$ then $R(\phi)$, the essential range of $\phi$, lies entirely inside or entirely outside $A$.

Proof. Lemma 1 says that $\phi(\theta)-\lambda=\alpha(\lambda) e^{L(\lambda, o)}$ where $L(\lambda, \theta)$ is continuous in $\lambda$ for each $\theta \in N^{\prime}$. For each $\theta$ the index (winding number) of $\Lambda$ with respect to $\phi(\theta)$ is the index of $-\alpha(\lambda)$ with respect to the origin, and so is independent of $\theta$. But the index is 1 if $\phi(\theta)$ is inside $A$ and 0 if $\phi(\theta)$ is outside $A$, and this establishes the lemma.

LeMma 3. If $\Lambda$ is a simple closed curve disjoint from $\sigma\left(T_{\phi}\right)$ and such that $R(\phi)$ lies entirely outside $\Lambda$, then $\sigma\left(T_{\phi}\right)$ lies entirely outside $\Lambda$.

Proof. Write

$$
\phi(\theta)-\lambda=e^{L(\lambda, \theta)+\log \alpha(\lambda)} \quad \lambda \in \Lambda, \theta \in N^{\prime}
$$


where $\log \alpha(\lambda)$ denotes a continuous logarithm of $\alpha(\lambda)$. This exists since $\alpha(\lambda)$ has index zero. Let $d \mu_{z}$ be the Borel measure on $A$ which solves the interior Dirichlet problem, i.e., if $f$ is a continuous function on $\Lambda$ then $\int f(\lambda) d \mu_{z}(\lambda)$ is the value at the point $z$ inside $\Lambda$ of the function harmonic inside $\Lambda$, continuous on the union of $\Lambda$ and its inside, and equal to $f$ on $A$. Now $L(\lambda, \theta)+\log \alpha(\lambda)$ is (for fixed $\left.\theta \in N^{\prime}\right)$ a continuous logarithm of $\phi(\theta)-\lambda$. Since $\phi(\theta)$ is outside $\Lambda$ this can be extended to a continuous logarithm of $\phi(\theta)-z$ for $z$ inside $\Lambda$. The extension is a harmonic function, so

$$
\int[L(\lambda, \theta)+\log \alpha(\lambda)] d \mu_{z}(\lambda)
$$

is the value of the extension at $z$. Consequently

$$
\phi(\theta)-\boldsymbol{z}=e^{\int[L(\lambda, \theta)+\log \alpha(\lambda)] d \mu_{z}(\lambda)} .
$$

The integral $I(\theta)=\int L(\lambda, \theta) d \mu_{\gamma_{2}}(\lambda)$ is a pointwise integral, i.e., for each $\theta, L(\lambda, \theta)$ is a Borel measurable function of $\lambda$ and $I(\theta)$ is its integral. We prefer to think of it as a weak integral, i.e., $I$ is the unique $L_{2}$ function which satisfies, for all $u \in L_{2}$,

$$
(I, u)=\int(L(\lambda, \theta), u(\theta)) d \mu_{z}(\lambda)
$$

This identity follows from Fubini's theorem. If we use the fact that $L(\lambda, \theta)=l(\lambda, \theta)$ a.e. for each $\lambda$, we can write (5) as

$$
\begin{aligned}
\phi(\theta)-z= & e^{\int \log \alpha(\lambda) d \mu_{z}(\lambda)} e^{\int \log \left|\phi_{+}(\lambda)\right| d \mu_{z}(\lambda)+i \int\left[\log \left|\phi_{+}(\lambda)\right|\right] \sim d \mu_{z}(\lambda)} \\
& \cdot e^{\int \log \left|\phi_{-}(\lambda)\right| d \mu_{z}(\lambda)-i \int\left[\log \left|\phi_{-}(\lambda)\right|\right] \sim d \mu_{z}(\lambda)}
\end{aligned}
$$

where all integrals are weak integrals. Now $\sim$ commutes with integration respect to $d \mu_{z}(\lambda)$; this follows from the definition of $\sim$ in terms of Fourier coefficients. Thus if we set

$$
\begin{aligned}
A & =e^{\int \log \alpha(\lambda) d \mu_{z}(\lambda)} \\
t_{+} & =\int \log \left|\phi_{+}(\lambda)\right| d \mu_{z}(\lambda) \\
t_{-} & =\int \log \left|\phi_{-}(\lambda)\right| d \mu_{z}(\lambda)
\end{aligned}
$$

we have

$$
\phi-z=A e^{t_{+}+\tilde{\mathrm{t}}_{+}} e^{t_{-}-\tilde{\mathrm{t}}_{-}}
$$


We shall show that this factorization exhibits the invertibility of $T_{\phi}-z$. Set

$$
\phi_{+}=A e^{t_{+}+\tilde{\mathrm{t}}_{+}}, \quad \phi_{-}=e^{t_{-}-\tilde{\mathrm{t}}_{-}} .
$$

We must verify that $\phi_{+}^{ \pm 1} \in H_{2}$, that $\phi_{-}^{ \pm 1} \in \bar{H}_{2}$, and that the map $f \rightarrow P f$ is bounded in $L_{2}\left(\left|\phi_{-}\right|^{2} d \theta\right)$.

The following fact is crucial. If $w_{1}, w_{2} \geqq 0$ satisfy

$$
\int|P f|{ }^{2} w_{i} d \theta \leqq M \int|f|{ }^{2} w_{i} d \theta
$$

for all $f \in L_{\infty}$, and $w=w_{1}^{\alpha} w_{2}^{1-\alpha}(0 \leqq \alpha \leqq 1)$, then also

$$
\int|P f|^{2} w d \theta \leqq M \int|f|^{2} w d \theta
$$

This follows from an interpolation theorem first proved for general operators and weight functions by Stein ([4], Theorem 2). We shall need an extension of this theorem to families of weight functions, and for convenience we state this extension together with another little fact as,

Sublemma. Assume $\lambda \rightarrow r(\lambda, \theta)$ is continuous from the compact set $\Lambda$ to real $L_{2}$ and such that for all $\lambda$

$$
\int e^{r(\lambda, \theta)} d \theta \leqq K
$$

Let $\mu$ be a nonnegative Borel measure on $\Lambda$ with $\mu(\Lambda)=1$. Then

$$
\int e^{\int r(\lambda, \theta) d \mu(\lambda)} d \theta \leqq K
$$

If in addition

$$
\int|P f|^{2} e^{r(\lambda, \theta)} d \theta \leqq M \int|f|^{2} e^{r(\lambda, \theta)} d \theta
$$

for all $f \in L_{\infty}$, then also

$$
\int|P f|^{2} e^{\int r(\lambda, \theta) d \mu(\lambda)} d \theta \leqq M \int|f|^{2} e^{\int r(\lambda, \theta) d \mu(\lambda)} d \theta
$$

Suppose for the moment that this has been established. If we apply the first part of the sublemma to the four functions $\pm \log \left|\phi_{ \pm}(\lambda)\right|^{2}$ and recall that by continuity the norms $\left\|\phi_{ \pm}(\lambda)^{ \pm 1}\right\|_{2}$ are uniformly bounded on $\Lambda$, we conclude that

$$
e^{ \pm t_{ \pm}}=e^{\int \log \left|\phi_{ \pm}(\lambda)\right| \pm 1_{\alpha \mu_{z}(\lambda)}}
$$

belong to $L_{2}$, and so $\phi_{+}^{ \pm 1} \in H_{2}$ and $\phi_{-}^{ \pm 1} \in \bar{H}_{2}$. Next it follows from $\left(\mathrm{c}^{\prime}\right)$ 
of the criterion for invertibility and the fact that $T_{\varphi}-\lambda$ is invertible for each $\lambda \in \Lambda$ that

$$
\int|P f|^{2}\left|\phi_{-}(\lambda)\right|^{2} d \theta \leqq M \int|f|^{2}\left|\phi_{-}(\lambda)\right|^{2} d \theta
$$

for all $f \in L_{\infty} ; M$ can be chosen independently of $\lambda$ since $\Lambda$ is bounded away from $\sigma\left(T_{\phi}\right)$. (See (1).) Therefore, by the sublemma again,

$$
\int|P f|^{2} e^{2 t}-d \theta \leqq M \int|f|^{2} e^{2 t}-d \theta,
$$

i.e., $f \rightarrow P f$ is bounded in $L_{2}\left(\left|\phi_{-}\right|^{2} d \theta\right)$. This concludes the proof of invertibility of $T_{\phi}-z$. Since $T_{\phi}-z$ is invertible for any $z$ inside $\Lambda$ we conclude that $\sigma\left(T_{\phi}\right)$ lies entirely outside $A$.

It remains to prove the sublemma. For each integer $n$ let $E_{n, i}$ $(i=1,2, \cdots)$ be a finite partition of $\Lambda$ into Borel sets so that

$$
\left\|r(\lambda, \theta)-r\left(\lambda^{\prime}, \theta\right)\right\|_{2}<\frac{1}{n}
$$

if $\lambda, \lambda^{\prime}$ belong to the same $E_{n, i}$. Choose points $\lambda_{n, i} \in E_{n, i}$ and set

$$
\begin{aligned}
w_{n} & =\exp \left\{\sum_{i} r\left(\lambda_{n, i}, \theta\right) \mu\left(E_{n, i}\right)\right\} \\
w & =\exp \left\{\int r(\lambda, \theta) d \mu(\lambda)\right\} .
\end{aligned}
$$

It follows from (6) that $\log w_{n} \rightarrow \log w$ in $L_{2}$ and our problem is to justify various passages to the limit under the integral sign. It follows from Hölder's inequality that for each $n$ we have $\left\|w_{n}\right\|_{1} \leqq K$. There is a sequence $n^{\prime}$ so that $w_{n^{\prime}} \rightarrow w$ a.e., so Fatou's lemma gives $\|w\|_{1} \leqq K$. This is the first part of the sublemma.

The unextended interpolation theorem has a trivial generalization to arbitrary finite logarithmically convex combinations of weight functions. Since $0 \leqq \mu\left(E_{n i}\right) \leqq 1$ and $\sum_{i} \mu\left(E_{n, i}\right)=\mu(\Lambda)=1$ we can conclude that for each $n$

$$
\int|P f|^{2} w_{n} d \theta \leqq M \int|f|^{2} w_{n} d \theta
$$

A slight modification of this which also follows from the unextended interpolation theorem is

$$
\int|P f|^{2} w_{n}^{1-\varepsilon} w_{1}^{\varepsilon} d \theta \leqq M \int|f|^{2} w_{n}^{1-\varepsilon} w_{1}^{\varepsilon} d \theta
$$

for all $\varepsilon(0<\varepsilon<1 / 2), n, f$. (Here $w_{1}$ is just $w_{n}$ with $n=1$.) By Hölder's inequality $\left\|w_{n}^{1-\varepsilon} w_{1}^{\varepsilon}\right\|_{1} \leqq K$. This implies that $w_{n}^{1-2 \varepsilon}$ have uniformly bounded norm in $L_{p}\left(w_{1}^{\varepsilon} d \theta\right)$, where $p=(1-\varepsilon) /(1-2 \varepsilon)$. 
Since $f \in L_{\infty}$ the functions $|f|^{2} w_{n}^{1-2 \varepsilon}$ also have uniformly bounded norm. Since $p>1$ we can find a sequence $n^{\prime}$ so that $|f|^{2} w_{n^{\prime}}^{1-2 \varepsilon}$ converge weakly to a function in $L_{p}\left(w_{1}^{\varepsilon} d \theta\right)$. But $n^{\prime}$ has a subsequence $n^{\prime \prime}$ so that $|f|^{2} w_{n^{\prime \prime}}^{1-2 \varepsilon}$ converges a.e. to $\mid f_{\mid}^{\prime 2} w^{1-2 \varepsilon}$. It follows that

$$
|f|^{2} w_{n^{\prime}}^{1-2 \varepsilon} \rightarrow|f|^{2} w^{1-2 \varepsilon}
$$

weakly. The conjugate space of $L_{p}\left(w_{1}^{\varepsilon} d \theta\right)$ is $L_{q}\left(w_{1}^{\varepsilon} d \theta\right)$ where $q=(1-\varepsilon) / \varepsilon$. Since $w_{1}^{\varepsilon} \in L_{q}\left(w_{1}^{\varepsilon} d \theta\right)$ it follows from the weak convergence that

$$
\int|f|{ }^{2} w_{n^{\prime}}^{1-2 \varepsilon} w_{1}^{2 \varepsilon} d \theta \rightarrow \int|f|^{2} w^{1-2 \varepsilon} w_{1}^{2 \varepsilon} d \theta
$$

This holds of course if $n^{\prime}$ is replaced by any subsequence, in particular one such that $w_{n^{\prime \prime}} \rightarrow w$ a.e. Then (7) with $\varepsilon$ replaced by $2 \varepsilon$, (8), and Fatou's lemma give

$$
\int|P f|^{2} w^{1-2 \varepsilon} w_{1}^{2 \varepsilon} d \theta \leqq \int|f|^{2} w^{1-2 \varepsilon} w_{1}^{2 \varepsilon} d \theta
$$

Since $w^{1-2 \varepsilon} w_{1}^{2 \varepsilon} \leqq \max \left(w, w_{1}\right) \in L_{1}$ we can take the limit as $\varepsilon \rightarrow 0$ under the integral on the right, and apply Fatou's lemma to the integral on the left, to obtain the final conclusion of the sublemma.

Now we are in a position to prove, without much more difficulty, that $\sigma\left(T_{\phi}\right)$ is connected. Suppose not. Then we can find a simple closed curve $\Lambda$, disjoint from $\sigma\left(T_{\phi}\right)$, so that a non-empty portion of $\sigma\left(T_{\phi}\right)$ lies inside $\Lambda$ and a non-empty portion of $\sigma\left(T_{\phi}\right)$ lies outside $\Lambda$. Call these portions $\sigma_{1}$ and $\sigma_{2}$ respectively. By Lemmas 2 and 3, $R(\phi)$ lies entirely inside $A$. Let $\Gamma_{\varepsilon}$ be a simple closed curve surrounding a non-empty portion $\sigma_{3}$ of $\sigma_{2}$ and such that each point of $\Gamma_{\varepsilon}$ is within $\varepsilon$ of $\sigma$. Since $\sigma_{2}$ is contained in the convex hull of $R(\phi)$ (in fact all of $\sigma\left(T_{\phi}\right)$ is; this will be explained in a moment) $\Gamma_{\varepsilon}$ will be contained in the convex hull of $A$ if $\varepsilon$ is sufficiently small. Thus of the three possibilities for disjoint simple closed curves ( $\Lambda$ and $\Gamma_{\varepsilon}$ will be disjoint is $\varepsilon$ is small enough),

$$
\begin{aligned}
& \Lambda \text { inside } \Gamma_{\varepsilon} \\
& \Gamma_{\varepsilon} \text { inside } \Lambda \\
& \Gamma_{\varepsilon}, \Lambda \text { have disjoint insides, }
\end{aligned}
$$

the first is eliminated since $\Gamma_{\varepsilon}$ is contained in the convex hull of $\Lambda$, the second is eliminated since $\sigma_{3}$ lies entirely outside $A$, and the third is eliminated by Lemma 3: since $R(\phi)$ lies outside $\Gamma_{3}$ so does $\sigma\left(T_{\phi}\right)$. The assumption that $\sigma\left(T_{\phi}\right)$ is disconnected has led to a contradiction.

It remains to see why $\sigma\left(T_{\phi}\right)$ is contained in the convex hull of $R(\phi)$. It suffices to show that $T_{\phi}$ is invertible if $R(\phi)$ is contained in an open angle of opening less than $\pi$ with vertex 0 , and since 
invertibility of $T_{\phi}$ is not destroyed by multiplying $\phi$ by a nonzero constant we may assume that this angle has the positive real axis as bisector. But then for sufficiently small $\varepsilon$ we shall have $\|1-\varepsilon \phi\|_{\infty}<1$, i.e. $\left\|I-\varepsilon T_{\phi}\right\|<1$, and this implies $T_{\phi}$ is invertible.

\section{REFERENCES}

1. A. Devinatz, Toeplitz operators on $\mathrm{H}^{2}$ spaces, Trans. Amer. Math. Soc., to appear. 2. P. R. Halmos, A glimpse into Hilbert space, article in "A Survey of Modern Mathematics," Wiley, 1963.

3. H. Helson and G. Szegö, A problem in prediction theory, Annali di Mat., 41 (1960), $107-138$.

4. E. M. Stein, Interpolation of linear operators, Trans. Amer. Math. Soc., 83 (1956), 482-492.

5. H. Widom, Inversion of Toeplitz matrices II, Ill. J. Math., 4 (1960), 88-99.

6. A. Zygmund, Trigonometric Series, vol. I, Cambridge, 1959.

CORNELL UNIVERSITY 



\section{PACIFIC JOURNAL OF MATHEMATICS}

\section{EDITORS}

Robert Osserman

Stanford University

Stanford, California

M. G. Arsove

University of Washington

Seattle 5 , Washington
J. DugundjI

University of Southern Califorma: Los Angeles 7, California

Lowell J. Paige

University of California

Los Angeles 24, California

\section{ASSOCIATE EDITORS}

E. F. BECKENBACH

B. H. NeumanN

F. WOLF

K. YosIDA

\section{SUPPORTING INSTITUTIONS}

UNIVERSITY OF BRITISH COLUMBIA

CALIFORNIA INSTITUTE OF TECHNOLOGY

UNIVERSITY OF CALIFORNIA

MONTANA STATE UNIVERSITY

UNIVERSITY OF NEVADA

NEW MEXICO STATE UNIVERSITY

OREGON STATE UNIVERSITY

UNIVERSITY OF OREGON

OSAKA UNIVERSITY

UNIVERSITY OF SOUTHERN CALIFORNIA
STANFORD UNIVERSITY

UNIVERSITY OF TOKYO

UNIVERSITY OF UTAH

WASHINGTON STATE UNIVERSITY

UNIVERSITY OF WASHINGTON

AMERICAN MATHEMATICAL SOCIETY CALIFORNIA RESEARCH CORPORATION SPACE TECHNOLOGY LABORATORIES NAVAL ORDNANCE TEST STATION 


\section{Pacific Journal of Mathematics}

\section{Vol. 14, No. 1 \\ May, 1964}

Richard Arens, Normal form for a Pfaffian .........................

Charles Vernon Coffman, Non-linear differential equations on cones in Banach

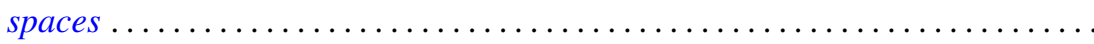

Ralph DeMarr, Order convergence in linear topological spaces ..............

Peter Larkin Duren, On the spectrum of a Toeplitz operator ................

Robert E. Edwards, Endomorphisms of function-spaces which leave stable all

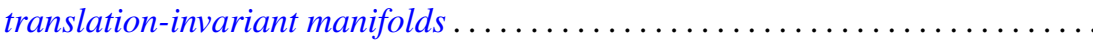

Erik Maurice Ellentuck, Infinite products of isols . . . . . . . . . . . . . . . . 49

William James Firey, Some applications of means of convex bodies . . . . . . . . 53

Haim Gaifman, Concerning measures on Boolean algebras ............. 61

Richard Carl Gilbert, Extremal spectral functions of a symmetric operator. . . . . . 75

Ronald Lewis Graham, On finite sums of reciprocals of distinct nth powers ..... 85

Hwa Suk Hahn, On the relative growth of differences of partition functions ...... 93

Isidore Isaac Hirschman, Jr., Extreme eigen values of Toeplitz forms associated

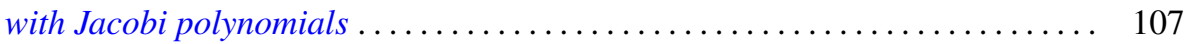

Chen-jung Hsu, Remarks on certain almost product spaces . . . . . . . . . . . 163

George Seth Innis, Jr., Some reproducing kernels for the unit disk . . . . . . . . . 177

Ronald Jacobowitz, Multiplicativity of the local Hilbert symbol . . . . . . . . . . . 187

Paul Joseph Kelly, On some mappings related to graphs ................. 191

William A. Kirk, On curvature of a metric space at a point . . . . . . . . . . . . 195

G. J. Kurowski, On the convergence of semi-discrete analytic functions . . . . . . . 199

Richard George Laatsch, Extensions of subadditive functions . . . . . . . . . . . 209

V. Marić, On some properties of solutions of $\Delta \psi+A\left(r^{2}\right) X \nabla \psi+C\left(r^{2}\right) \psi=0 \ldots 217$

William H. Mills, Polynomials with minimal value sets . . . . . . . . . . . 225

George James Minty, Jr., On the monotonicity of the gradient of a convex

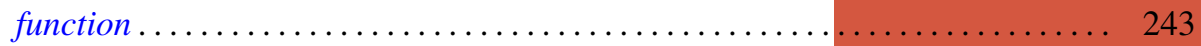

George James Minty, Jr., On the solvability of nonlinear functional equations of 'monotonic' type ................................... 249

J. B. Muskat, On the solvability of $x^{e} \equiv e(\bmod p) \ldots \ldots \ldots \ldots \ldots \ldots \ldots \ldots . \ldots \ldots$

Zeev Nehari, On an inequality of $P . R$. Bessack ................... 261

Raymond Moos Redheffer and Ernst Gabor Straus, Degenerate elliptic

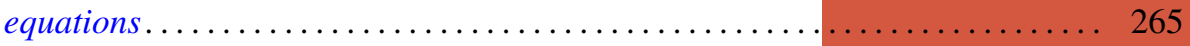

Abraham Robinson, On generalized limits and linear functionals . . . . . . . . . 269

Bernard W. Roos, On a class of singular second order differential equations with a

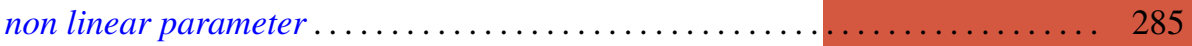

Tôru Saitô, Ordered completely regular semigroups . . . . . . . . . . . . . . . . 295

Edward Silverman, A problem of least area ....................... 309

Robert C. Sine, Spectral decomposition of a class of operators . . . . . . . . . 333

Jonathan Dean Swift, Chains and graphs of Ostrom planes . . . . . . . . . . . 353

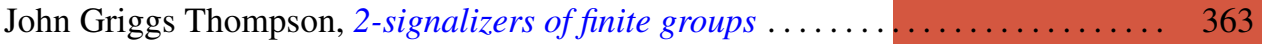

Harold Widom, On the spectrum of a Toeplitz operator . . . . . . . . . . . . . 365 